nahme zu beachten. Metformin und Acarbose haben oft gastrointestinale Nebeneffekte. Von den Gliptinen ist nur Sitagliptin (etwa Xelevia ${ }^{\circledR}$ ) zur Monotherapie zugelassen.

In Kombinationstherapien, etwa mit Metformin, kommen als Glitazonersatz ein Gliptin, ein Inkretinmimetikum, $\mathrm{SH}$, Acarbose (bis zu einer glomerulären Filtrationsrate von etwa $25 \mathrm{ml} / \mathrm{min}$ ), Insulin oder Insulin plus Sitagliptin in Betracht. Gliptine und GLP-1-Mimetika haben kein erhöhtes Hypoglykämierisiko, wenn sie nicht mit insulinotropen Präparaten kombiniert werden. Sitagliptin gibt es bereits als Fixkombination mit Metformin (etwa Velmetia $^{\circledR}$ ). Sitagliptin ist auch das einzige Gliptin mit Zulassung zur Kombination mit Insulin. Beispiel einer Umstellungsentscheidung ist ein 54
Jahre alter Patient: Body-Mass-Index 36, behandelte Hypertonie und Hypercholesterinämie, herzkrank, Raucher, Niere und Leber in Ordnung, Typ2-Diabetes seit acht Jahren. Mit täglich $8 \mathrm{mg}$ Rosiglitazon und $1700 \mathrm{mg}$ Metformin betrug der $\mathrm{HbA}_{1 \mathrm{c}}$-Wert 7,4\%. Insulin lehnte er ab; es wäre wegen des Gewichts auch zweite Wahl gewesen. Die Metformindosis wurde auf zweimal $1 \mathrm{~g}$ angehoben und der Patient nochmals geschult. Als Zusatz kamen wegen der Gewichts- und Complianceprobleme Gliptine und GLP-1-Analoga in Betracht. Da der Patient nicht injizieren wollte, entschied sich der Diabetologe für ein Gliptin.

$h b r$

Symposium bei der DDG-DAG-Tagung, 5.11.2010

in Berlin, Veranstalter: Berlin Chemie

\title{
Blutzuckerselbstmessung
}

\section{Sie ist nur so gut wie das Messgerät}

- Für eine optimale Blutzuckerkontrolle gilt es, die Balance zwischen Hyper- und Hypoglykämie zu halten. Besonders bei intensivierter konventioneller Insulintherapie (ICT) ist hierfür die Blutzuckerselbstmessung (BZSM) unerlässlich.

Zwei Zustände können bei Diabetespatienten mit einer Insulintherapie schwerwiegende Ereignisse auslösen: die Hyperglykämie, deren Vermeidung zu weniger diabetesbedingten Komplikationen wie Herzinfarkt, Amputation und Retinopathie führt, und die Hypoglykämie. Schwere Hypoglykämien führten zu einem Anstieg der Hospitalisierungssterblichkeit um $80 \%$. Dazu verwies

\section{Kurz notiert}

\section{Messgerät plus Ratgeber}

Nun ist das Blutzuckermesssystem OneTouch ${ }^{\circledR}$ Vita ${ }^{\circledR}$ von LifeScan auch als Sonderedition erhältlich. Neben Traubenzucker zur schnellen Blutzuckerregulierung ist der Produktpackung auch der handliche Ratgeber „Kalorien mundgerecht für unterwegs" beigelegt. Dieser gibt Aufschluss über Kalorien und Fettgehalt bestimmter Nahrungsmittel und BE-Angaben. Diabetesschwerpunktpraxen sowie diabetologisch tätige Allgemeinmediziner können das Set über den LifeScan-Außendienst anfordern.

Weitere Informationen zu OneTouch ${ }^{\circledR}$-Blutzuckermesssystemen und Serviceleistungen von LifeScan erhalten Sie beim LifeScan-Kundenservice unter der gebührenfreien Rufnummer 0800 - 7077007.
Dr. Hans-Martin Reuter, Jena, auf Studienergebnisse, die beim ADA-Kongress 2010 in Orlando vorgestellt wurden. Zudem hätten Patienten mit Hypoglykämien ein um 79\% erhöhtes Risiko für kardiovaskuläre Ereignisse im Vergleich zu Patienten ohne Hypoglykämien. Auch mehren sich Hinweise, dass schwere Hypoglykämien eine Demenzentwicklung begünstigen können.,,Je weiter $\operatorname{der} \mathrm{HbA}_{1 \mathrm{c}}$ abgesenkt wird, umso höher ist das Hypoglykämierisiko“, sagte Reuter.

Besonders bei Patienten mit ICT ist es daher wichtig, den individuellen Schnittpunkt zwischen optimalem Schutz durch $\mathrm{HbA}_{1 \mathrm{c}}$-Senkung und minimalem Hypoglykämierisiko zu finden. Hier ist die BZSM sehr wichtig. „Je besser und konsequenter ein Patient seinen Blutzucker misst, umso besser sind seine $\mathrm{HbA}_{1 \mathrm{c}}$-Werte und desto geringer ist das Hypoglykämierisiko“, so Reuter.

Patienten können jedoch nur so gut messen, wie die Blutzuckermessgeräte zuverlässige Ergebnisse liefern. In einer Untersuchung wurden 27 Messgeräte geprüft [1]. Es stellte sich heraus, dass mehr als 40\% der evaluierten Geräte Ungenauigkeiten hatten. Bei vielen Herstellern besteht hier ein hoher Handlungsbedarf. Denn die Patienten können nur wesentlich zu einer optimalen Blutzuckereinstellung beitragen, wenn die Geräte exakte Ergebnisse liefern, wie es für jene der ONETOUCH ${ }^{\circledR}$-Serie belegt wurde.

cvs

1. Freckmann G et al. Diabetes Technology \& Therapeutics 2010;12(3).

Veranstaltung bei der DDG-DAG-Tagung, 5.11.2010 in Berlin, Veranstalter: LifeScan 